\title{
An endovascular canine stroke model: middle cerebral artery occlusion with autologous clots followed by ipsilateral internal carotid artery blockade
}

\author{
Qing-Quan Zu*, Sheng Liu*, Xiao-Quan Xu, Shan-Shan Lu, Lei Sun and Hai-Bin Shi
}

Stroke is one of the leading causes of death worldwide and the main reason for long-term disability. An appropriate animal model of stroke is urgently required for understanding the exact pathophysiological mechanism of stroke and testing any new therapeutic regimen. Our work aimed to establish a canine stroke model occluding the middle cerebral artery (MCA) and blocking the ipsilateral internal carotid artery (ICA), and to assess the infarct lesions by magnetic resonance imaging. The stroke model was generated by injecting two autologous clots into each MCA, followed by $2-h$ ipsilateral ICA blockade (illCAB) using a catheter in 15 healthy adult beagles. Outcome measurements included 24-h and 7-day postocclusion T2-weighted imaging (T2WI)-based infarct volume calculation. In addition, pial collateral score, canine neurobehavioral score and histopathologic results were documented. Out of 15 dogs, 12 with successful MCA occlusion (MCAO) and illCAB survived 7 days without complications or casualties and MCA were reperfused at 7 days after occlusion. High signal intensity in the basal ganglia and cerebral cortex on T2WI was initially observed in each dog at $6 \mathrm{~h}$ after the procedure. The mean percentage hemispherical infarct volume corrected for edema in all dogs on T2WI at $24 \mathrm{~h}$ after occlusion was $12.99 \pm 1.57 \%$, and the degree of variability was $12.08 \%$. The infarct volumes at $24 \mathrm{~h}$ after occlusion correlated with pial collateral scores and canine neurobehavioral scores well. This canine stroke model with combined MCAO and illCAB reported here were proven to be highly feasible and reproducible.

Laboratory Investigation (2013) 93, 760-767; doi:10.1038/labinvest.2013.65; published online 6 May 2013

KEYWORDS: angiography; beagle; embolism; model; stroke

Stroke is currently the leading cause of serious long-term disability and the third leading cause of death in the world. ${ }^{1-4}$ The understanding of pathophysiology of stroke and testing any therapeutic regimen make an ideal and reproducible model of stroke necessary. Considering limitations in cerebral infarction animal models generated by other groups (eg, different cerebrovascular anatomy between small animal and human being, translational barriers between experimental and clinical studies, and ethical and economic issues in nonhuman primates), this canine ischemic stroke model has emerged as an ideal option in clinical research ${ }^{1,2,5-10}$ with many advantages. First, the anatomic features of the canine brain are more close to human brain compared with those in other small animals. Second, the cerebrovascular structure and brain volume of canines are suitable for routine radiological methodology, which permits a minimally invasive approach, a direct and targeted arterial embolization and real-time visualization of occlusion. ${ }^{1,2}$ In addition, magnetic resonance imaging (MRI) provides an accurate way to follow the progression of stroke models in serial images. ${ }^{10-13}$

However, strokes in humans and canine models are highly heterogeneous both clinically and radiographically. ${ }^{10,13}$ In human strokes, one vessel blockade often results in large or consecutive ischemia lesions. However, segmental occlusion of one single vessel (eg, middle cerebral artery (MCA)) in dogs often cannot produce significant defects ${ }^{10,14,15}$ because of compensation due to their leptomeningeal collateral circulation. Abundant collateral blood flow could develop from the distal area to the occluded locus before an irreversible ischemic lesion could happen, ${ }^{2,10,16-18}$ which results in a major mismatch between the size of infarct area

Department of Radiology, The First Affiliated Hospital of Nanjing Medical University, Gulou District, Nanjing, Jiangsu, China

Correspondence: Professor H-B Shi, MD, PhD, Department of Radiology, The First Affiliated Hospital of Nanjing Medical University, 300 Guangzhou Road, Gulou District, Nanjing, Jiangsu 210029, China.

*These authors contributed equally to this work.

Received 1 January 2013; revised 18 March 2013; accepted 2 April 2013 
and the territory of the vessel occlusion. Lipsanen and Jolkkone $^{19}$ reported that consecutive lesions could be produced by MCA occlusion (MCAO) followed by ipsilateral intracranial blood supply artery blockade in rat stroke models.

Herein, we hypothesized that relative large infarct lesions can be produced by endovascular method of MCAO followed by ilICAB, which can reduce the leptomeningeal collateral blood compensation and lower the chance of premature recanalization simultaneously and eventually mimic the pathology of humans' cerebral infarction. Thus, this study would describe how to generate a relatively large area stroke model in beagles using two autologous clots by segmental occlusion of the distal and proximal MCA followed by 2-h ilICAB with 5-French catheter, and identify and serially assess infarct lesions using T2-weighted imaging (T2WI).

\section{MATERIALS AND METHODS}

All animal care and experimental procedures were performed in accordance with regulations specified by the Guide for the Care and Use of Laboratory Animals (NIH Publication No. 85-23, revised 1996) and the protocols approved by the Institutional Animal Care and Use Committee of Nanjing Medical University.

\section{Endovascular Canine MCAO}

Fifteen male beagles $(13-15 \mathrm{~kg}$ ) from the Laboratory Animal Center of Nanjing Medical University were acclimatized to our animal facility for one day before experiments. The methods of anesthesia, monitoring and maintaining the canine physiologic parameters within normal ranges were optimized as described in our previous study. ${ }^{10}$

Right common femoral artery and left femoral vein accesses were obtained by using 5-French sheaths (Terumo Medical Corporation, Tokyo, Japan). Clots were prepared as described previously. ${ }^{10}$ Notably, the thread-like clots were cut into about 1.4 and $1.7 \mathrm{~mm}$ in diameter and $5 \mathrm{~mm}$ in length, and then were placed in a sterilized Petri dish containing normal saline. The animal was initially administered a bolus of $2500 \mathrm{U}$ of heparin ${ }^{18}$ and left femoral vein access was maintained with a saline drip $(2 \mathrm{ml} / \mathrm{min})$.

A 5-French vertebral catheter was inserted into cerebral vessels under fluoroscopic guidance (Axiom Artis, Siemens, Munchen, Germany). After a baseline arteriography was performed, the catheter was advanced into left ICA about $2 \mathrm{~cm}$ in the ascending part. Then, a clot with $1.4 \mathrm{~mm}$ in diameter was placed into a $2-\mathrm{ml}$ syringe filled with contrast agent (Omnipaque 300; GE Healthcare, USA). After a clot was injected into ICA, a $2-\mathrm{ml}$ syringe was replaced by a $5-\mathrm{ml}$ syringe filled with normal saline and the normal saline was injected into ICA slowly with intermittent pressure. A baseline image scan was performed to document whether the distal part of the M1 segment of MCA was occluded; if yes, then another clot with $1.7 \mathrm{~mm}$ in diameter would be injected to occlude the proximal part of the M1 segment (Figure 1).

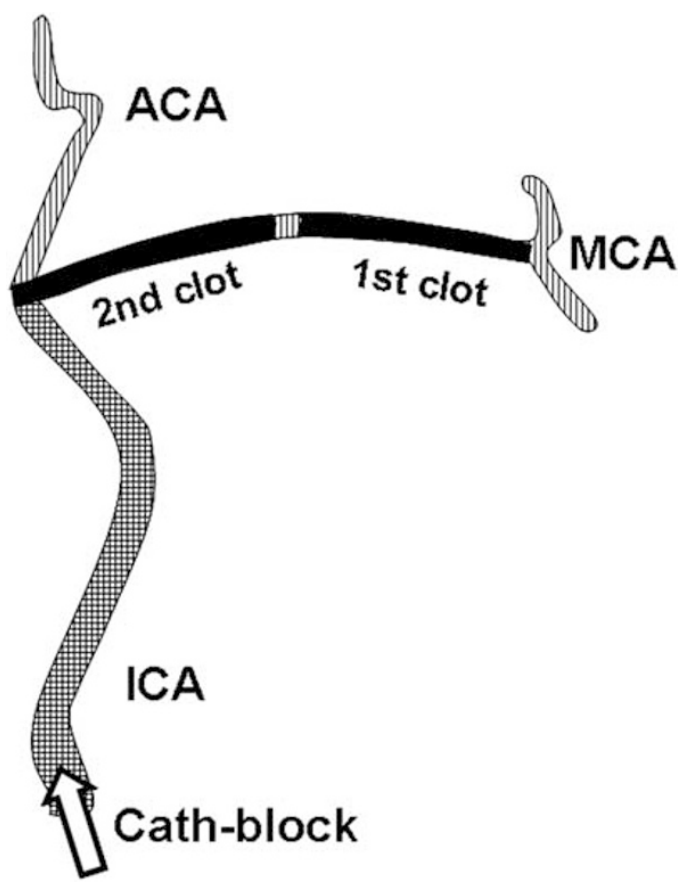

Figure 1 Ideograph of middle cerebral artery (MCA) occlusion in canine. ACA, anterior cerebral artery; cath-block, ICA was blocked by a 5-French catheter for $2 \mathrm{~h}$; ICA, internal carotid artery.

Angiography was performed to confirm the occlusion of the M1 segment and evaluate the leptomeningeal collateral recruitment. An 11-point scale was implemented in this study, which took into account the anatomic extent and transit time of leptomeningeal collateral circulations from the posterior and anterior cerebral arteries to the affected MCA territory ${ }^{18}$.

Incomplete $\mathrm{MCAO}$ in angiography was considered as the exclusion criteria. If MCAO was complete, the ipsilateral ICA would be blocked with the same catheter connected to pressurized normal saline for $2 \mathrm{~h}$. After that, we removed the catheter and sheath from the femoral artery, and compressed the insertion site to stop bleeding. Thereafter, animals were transported to the MRI suite after the procedure. The same endovascular procedure and followup angiography were repeated to document whether recanalization of the left MCA happens at $24 \mathrm{~h}$ and 7 days after embolization.

\section{MR Scanning}

Evaluation of the infarct lesion was accomplished in a 3.0-T whole-body MR scanner (Siemens Medical Solutions, Erlangen, Germany) using an 8-channel, transmit-receive extremity coil with a diameter of $15 \mathrm{~cm}$. The imaging protocols consisted of coronal T1-weighted (TR/TE 1390/11, matrix $320 \times 320)$ and T2-weighted $(\mathrm{TR} / \mathrm{TE} 5000 / 66$, matrix $320 \times 320$ ) sequences with 22 sections and non-skip $2-\mathrm{mm}$ section thickness. All dogs were scanned before operations to document baseline images. Then, MRIs were repeated at $3 \mathrm{~h}$, 
$6 \mathrm{~h}, 24 \mathrm{~h}$ and 7 days after MCAO and ilCIAB. Calculation of infarct volume was obtained from T2WI (24h and 7 days after occlusion) by computer-aided manual tracing of the infarct margin and the ipsilateral and the contralateral hemispheres. Correction for edema-induced midline shift in hemispherical volume was incorporated into infarct volume calculations as described previously. ${ }^{2,20}$

\section{Neurobehavioral Assessment}

Neurobehavioral assessment was performed by a neurologic interventional radiologist before the procedure at $24 \mathrm{~h}$ and 7 days following embolization. A simple 12-point canine neurobehavior scoring system (Table 1), including assessments of motor function, consciousness, head turning, circling and hemianopsia, was adapted for the canine ischemic stroke models. ${ }^{10}$

\section{Histological Examination}

Dogs were killed immediately with an intravenous overdose injection of pentobarbital solution $(80 \mathrm{mg} / \mathrm{kg})$ after all examinations. Necropsy was performed to isolate the brains. After gross examination, brains were frozen at $-20{ }^{\circ} \mathrm{C}$ for $30 \mathrm{~min}$. Four-millimeter-thick coronal sections were collected throughout the brain and bathed in 2\% TTC solution (Ruji Biology Technology Company, China) at $37^{\circ} \mathrm{C}$ in the dark room for $1 \mathrm{~h}$ and periodically agitated to ensure that

Table 1 Canine neurobehavior scoring system

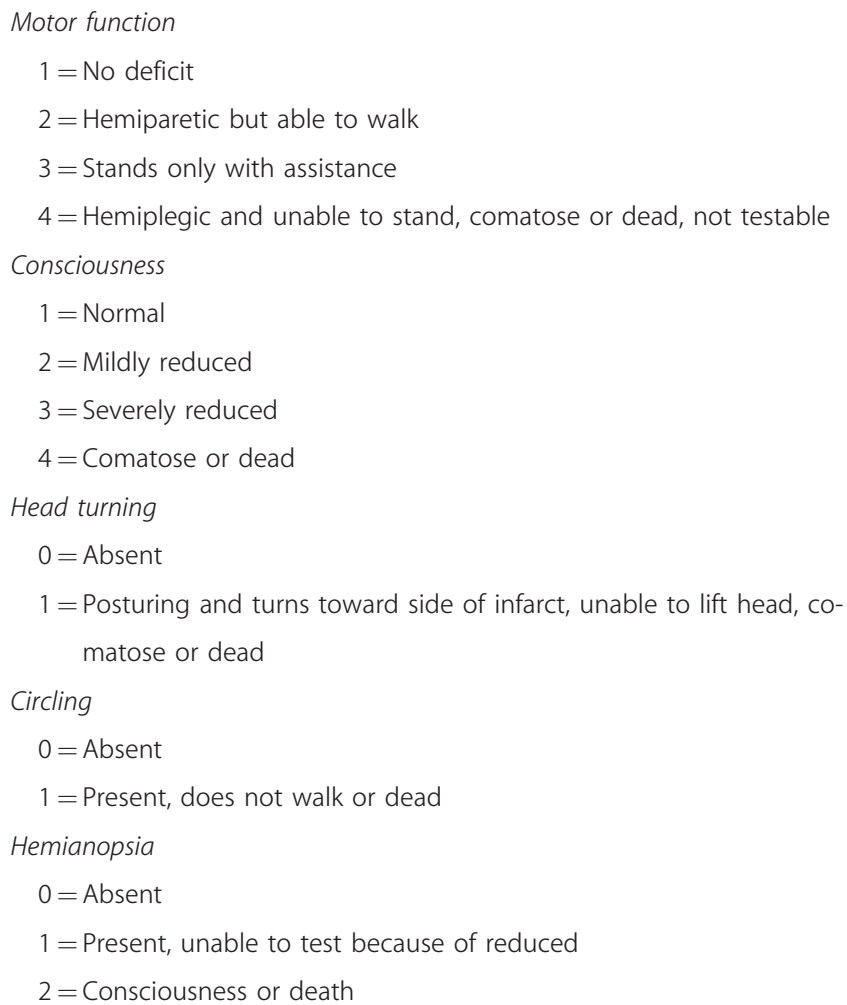

they were well stained. Subsequently, the stained slices were immersed in 10\% neutral-buffered formalin and embedded in paraffin after $72 \mathrm{~h}$ of fixation. Paraffin-embedded slices were cut into $5-\mu \mathrm{m}$-thick sections, HE-stained and mounted for histological analysis.

\section{Statistical Analysis}

The infract volumes of $24 \mathrm{~h}$ and 7 days were compared using a paired $t$-test. The neurological scores of $24 \mathrm{~h}$ and 7 days were compared using Wilcoxon's test. Pial collateral score and canine neurobehavioral score were correlated to infarct volume measured on 24-h T2WI using linear fit analysis. $P$-value $<0.05$ was considered statistically significant and SPSS 16.0 was used in all calculations.

\section{RESULTS \\ Angiographic Findings}

Twelve beagles with successful intended modeling survived after 7 days study. Three dogs with incomplete proximal MCA embolized were excluded for further analysis. The efficiency was $80 \%(12 / 15)$. In these animals, arteriograms showed successful MCAO and ilICAB immediately after embolization, no recanalization happened at $24 \mathrm{~h}$ and reperfusions were observed at 7 days after occlusion (Figure 2). Leptomeningeal collateral recruitment scores ranged from 3 to 5 points (mean $3.8 \pm 0.8$ points) at the time of occlusion (Supplementary Table 1). The experience of occluding the distal and proximal M1 segments of MCA by two clots was obtained from both previous work and pilot study.

\section{MR Imaging}

High signal intensity on T2WI was initially observed in all animals at $6 \mathrm{~h}$ after the ipsilateral MCAO, while the signal intensity of T2WI changed over time and the margins became more distinct (Figure 3). Each animal had ischemic lesions located in the basal ganglia and cerebral cortex.

The mean infarct volume on T2WI was 4173.23 \pm $603.92 \mathrm{~mm}^{3}$ at $24 \mathrm{~h}$ after the onset of stroke. The infarction volume on T2WI was $3273.21 \pm 616.05 \mathrm{~mm}^{3}$ at 7 days and significantly smaller than that at $24 \mathrm{~h}$ after stroke $(t=8.98$, $P<0.01)$. There was no hemorrhage or obvious midline shift of the brain identified. The mean percentage of hemispherical infarct volume corrected for edema in all dogs on T2WI at $24 \mathrm{~h}$ after occlusion was $12.99 \pm 1.57 \%$, and the degree of variability was $12.08 \%$. The infarct volumes corrected for edema at $24 \mathrm{~h}$ after occlusion were correlated with pial collateral scores negatively $\left(r^{2}=0.361, P=0.039\right)$ (Figure 4a).

\section{Neurobehavioral Scores}

All animals recovered from anesthesia and were enrolled in evaluating neurological deficits at $24 \mathrm{~h}$ and 7 days after occlusion. All dogs showed neurologic deficits, including reduced responsiveness, head turning and circling, contralateral hemiparesis, walking with or without assistance and 

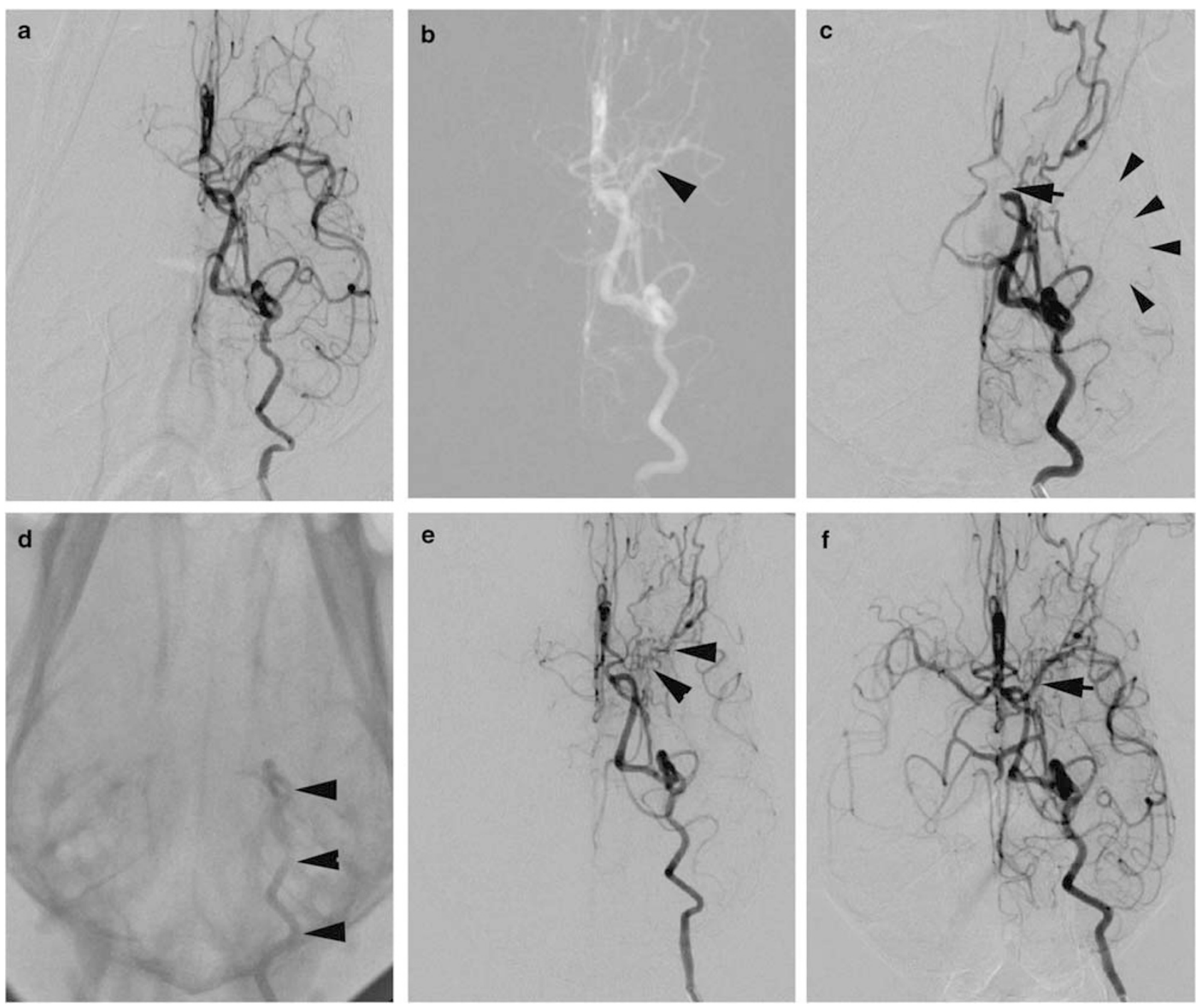

Figure 2 Minimally invasive approach to left middle cerebral artery (MCA) occlusion in beagle dog. The angiography pre-occlusion showed cerebrovascular architecture (a), immediately after occlusion (arrow) indicated that there was merely no collateral flow to the occluded territory (arrowhead) (c), $24 \mathrm{~h}$ after occlusion confirmed no recanalization (arrowhead) (e) and 7 days after embolization revealed reperfusion of MCA (arrow) (f). The first clot occluded distal M1 segment (arrowhead) (b). The stagnation of contrast agent in ipsilateral internal carotid artery (ICA) (arrowhead) indicated that the catheter could prohibit blood flow into cerebra (d).

hemianopsia (Supplementary Movie 1). The mean neurobehavior score was $6.08 \pm 1.0$ at $24 \mathrm{~h}$ after embolization. Canine neurobehavioral deficits improved with a decrease of $0-3$ points in neurobehavioral score during the follow-up days. The mean score was $4.42 \pm 0.51$ at the 7 th day after the procedure, and the statistical difference of neurobehavioral scores between $24 \mathrm{~h}$ and 7 days after MCAO was significant $(Z=-3.025, P=0.002)$. The infarct volumes corrected for edema at $24 \mathrm{~h}$ after occlusion were correlated with canine neurobehavioral scores positively $\left(r^{2}=0.422, \quad P=0.022\right)$ (Figure 4b).

\section{Pathological Findings}

Gross pathological study of the brain sections confirmed well-defined basal ganglia and cerebral cortex infarctions on the occluded side of animal brains without apparent hemorrhagic focus. There appeared to be liquefied areas at infarction cores and clear delineation between infract and normal brain with TTC staining. These findings matched with MRI results well. Microscopic readouts showed clear margin infarcts and neuronal cell loss, shrunken and pyknotic nuclei in infarcted areas (Figure 5).

\section{DISCUSSION}

In experimental studies of ischemic stroke, the efficiency and meaning of the model should be considered carefully. Our study has demonstrated the high feasibility and reproducibility of an endovascular canine stroke model combined with two autologous blood clots generated by MCAO followed by catheter-assisted ilICAB, which make it a unique model that 

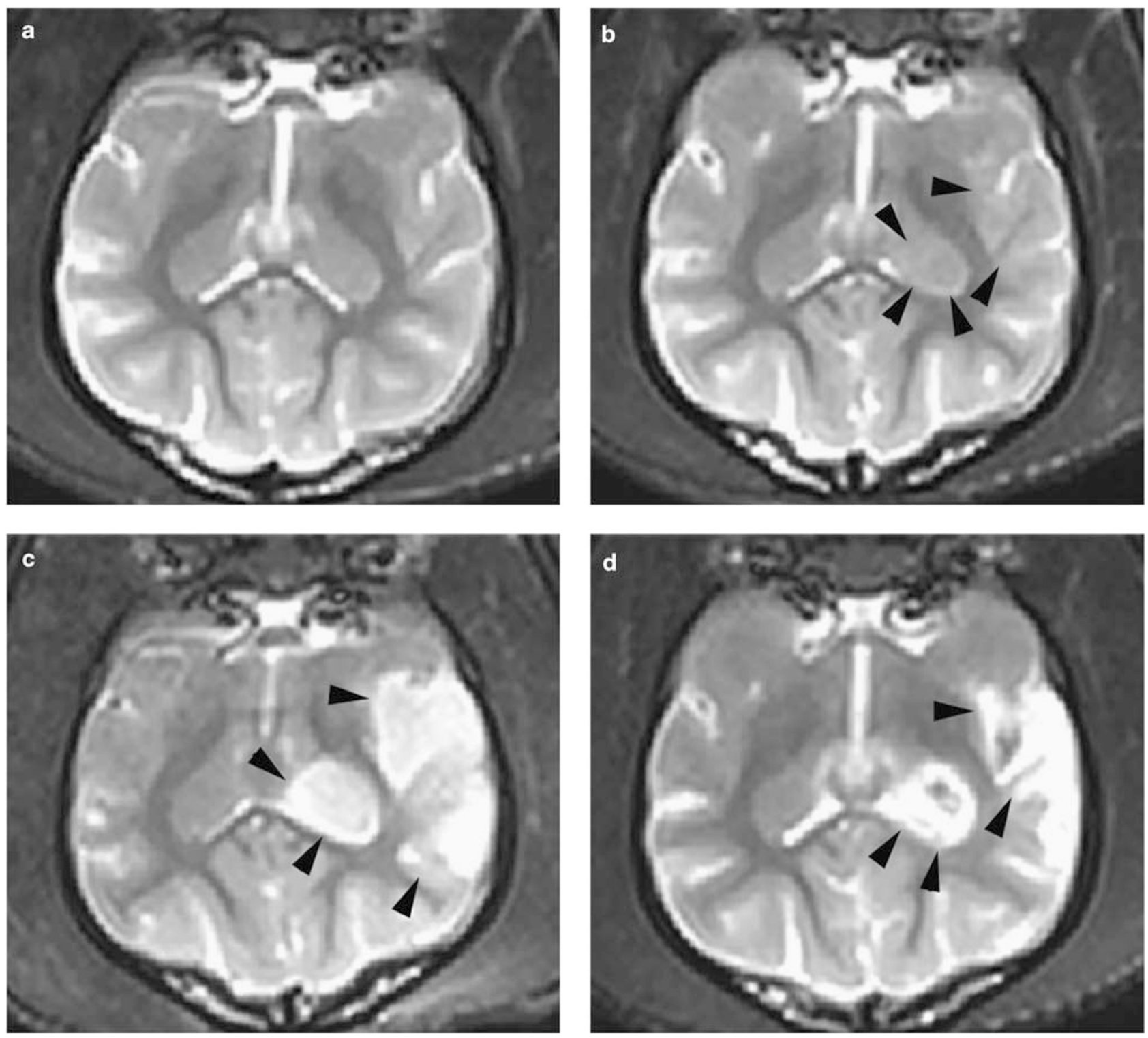

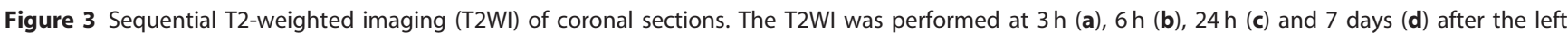
middle cerebral artery (MCA) occlusion, respectively. Negative finding was shown in T2WI within $3 \mathrm{~h}$ (a). High signal intensity areas were initially observed in the basal ganglia (arrowhead) and cerebral cortex (arrowhead) at $6 \mathrm{~h}$ after occlusion (b); high signal intensity became clear (arrowhead) at $24 \mathrm{~h} \mathrm{(c)} \mathrm{and} \mathrm{infarct} \mathrm{got} \mathrm{well-defined} \mathrm{(arrowhead)} \mathrm{at} \mathrm{the} \mathrm{seventh} \mathrm{day} \mathrm{(d)} \mathrm{in} \mathrm{the} \mathrm{same} \mathrm{lesions.}$

could highly mimic the common etiological factor of human ischemic stroke. Our model produce relatively large infarct volume benefiting from limiting the pial collateral blood supply, lower the risk of recanalization and survive for 7 days. Briefly, little variation in the brain architectures and the diameters of cerebral vessels in beagles, which are structurally similar to human, were the intrinsic factors, while the understanding of the pial collateral recruitment was the extrinsic factor of high modeling feasibility and reproducibility. Notably, the model had obvious changes on both radiologic manifestations and clinical features. It is worth using it as an ideal stroke model in the future translational research. At the same time, we have evaluated the pial collateral recruitment by real-time fluoroscopy and serially showed the progression of infarct lesions from $3 \mathrm{~h}$ to 7 days after occlusion of MCA on MRI. The infarct areas were located in the basal ganglia and cerebral cortex, consistent with the MCA supplied territory. We also found that the lesions were consecutive rather than sporadic.

Different anatomic and pathophysiological factors can conceivably contribute to different consequences of strokes. ${ }^{15}$ In studies of Rink et $a l^{2}$ and Christoforidis et al, ${ }^{18}$ coils, other than thrombus, were used to generate stroke models, which called into questions about the physiological relevance of the model and risk of vasospasm or perforation of intracranial arteries. Moreover, considering that ischemic infarct was 
usually caused by thrombi composed of platelets and/or fibrin in humans, the thrombin-induced clot may recapitulate an ideal stroke model. ${ }^{10,21}$ Several studies had described that the embolus of $5 \mathrm{~mm}$ in length and about
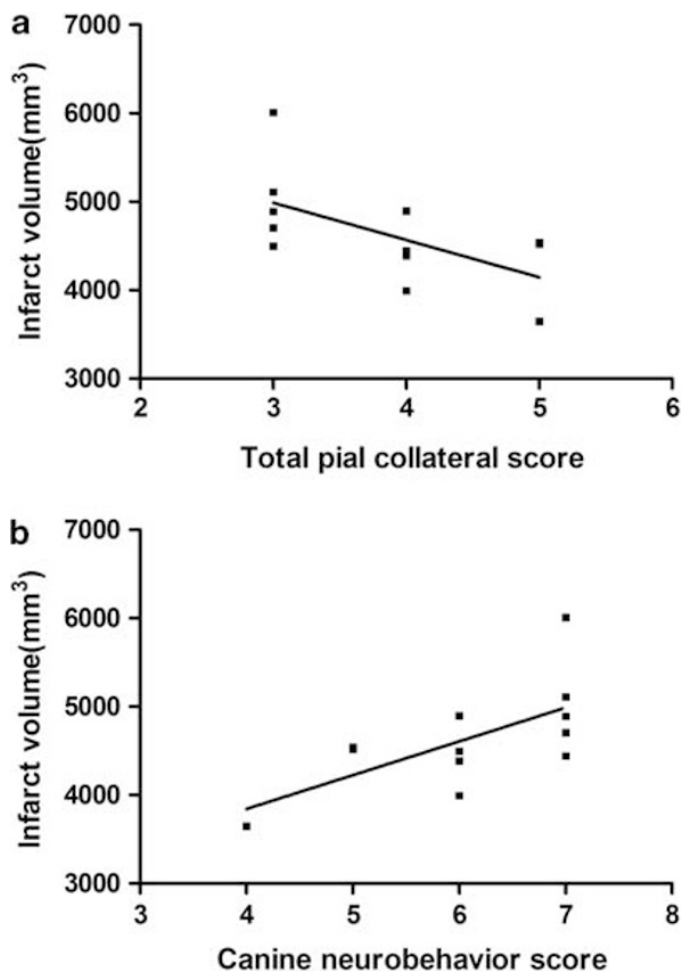

Figure 4 Bivariate linear fit for infarct volume versus pial collateral score and canine neurobehavioral score. The infarct volumes at $24 \mathrm{~h}$ after occlusion correlated with pial collateral scores negatively $\left(r^{2}=0.361\right.$, $P=0.039)(a)$ and canine neurobehavioral scores positively $\left(r^{2}=0.422\right.$, $P=0.022)(\mathbf{b})$.

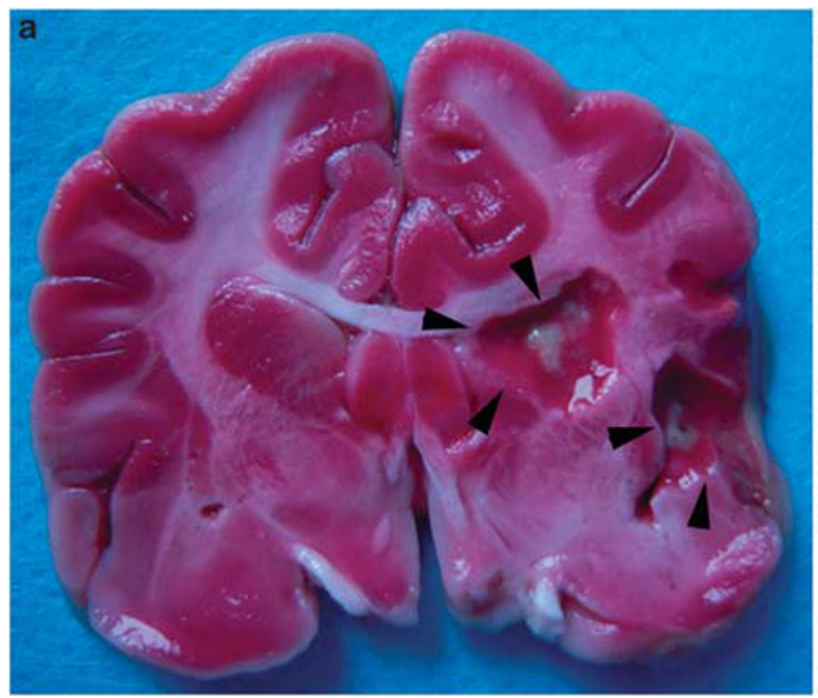

$1.7 \mathrm{~mm}$ in diameter went into the proximal segment of MCA via ICA delivery. ${ }^{1,10,22}$ While, in our pilot study, we observed that the clot with a diameter of $1.4 \mathrm{~mm}$ could lodge at the distal M1 segment and block blood to flow into the M2 segment. The M1 segment was about $1.0 \mathrm{~mm}$ in diameter and $13 \mathrm{~mm}$ in length in beagles (unpublished data). Thus, the clots of 1.4 and $1.7 \mathrm{~mm}$ in diameter were made intentionally to occlude the distal and proximal parts of the M1 segment, respectively. The angiograms showed complete occlusion and no-recanalization at $24 \mathrm{~h}$ after MCAO, which elucidated that the clots were size-suitable, stable, elastic and easy to inject through the catheter in this study. It is important to mention that there is a need to keep pushing the plunger with an even intermittent pressure until the clots lodged in the target vessels under fluoroscopy. Although the intrinsic fibrinolytic system of canine is more active than that of humans, ${ }^{18}$ it has been proven that MCAO combined with common carotid artery occlusion can produce consistent lesions. ${ }^{19}$ Here, we blocked the ipsilateral ICA with a catheter to delay blood flow transit time and attenuate the effect of intrinsic fibrinolytic system, which intended to avoid premature recanalization or migration of clots to obtain high reproducible results. Two hours of ilICAB has proven to be an optimal time duration to achieve our goal efficiently (unpublished data). On the seventh day, angiography confirmed recanalization of MCA and further studies will use this model to test potential transarterial therapeutic regimens in different phases of acute stroke.

In addition, the variability of infarction volumes was a little high in stroke models with MCAO reported by many other studies, which could result from the variation of the vascular anatomy and leptomeningeal collateral recruitment. ${ }^{18,23-26}$ In canine stroke models, pial collateral recruitment was reported to be influenced by the following

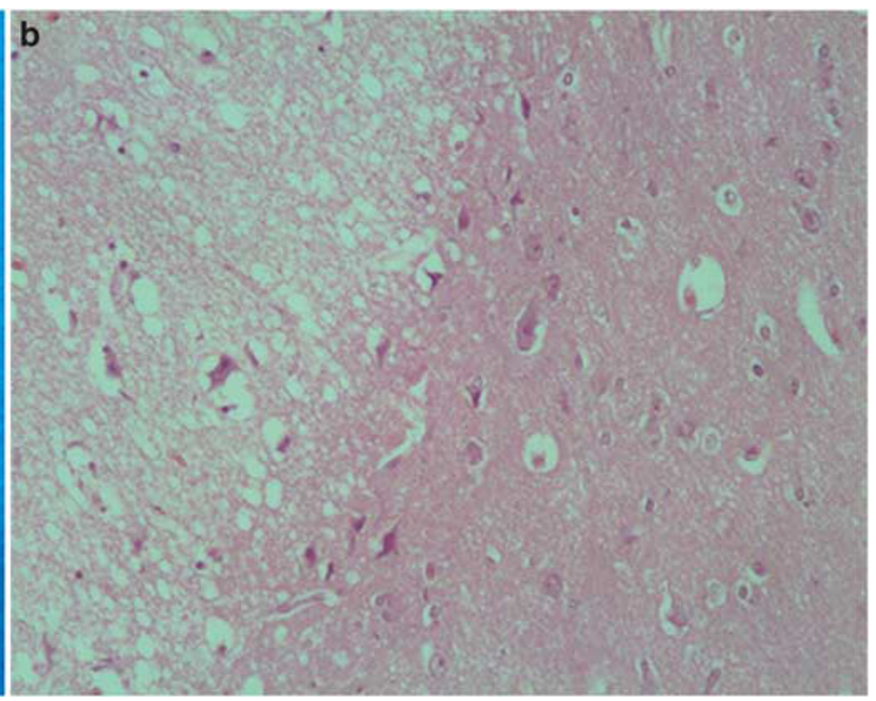

Figure 5 Pathological features of canine infarct. The brain after 2, 3, 5-triphenyltetrazolium chloride (TTC) staining demonstrated infarct lesions (arrowhead) and liquefied area at infarct cores (a). Microscopic view of the infracted area showed clearly distinguished edges between infracted and normal brain, prominent neuronal cell loss and nuclear karyolysis (hematoxylin and eosin (HE) staining, $\times 200)(\mathbf{b})$. 
factors: vascular dynamics, systemic blood pressure and so on. ${ }^{18,27}$ Besides, the occlusion site and the size of thrombus also had a certain impact on the infarct volume. ${ }^{15,24}$ In our previous study, we observed that the connections of pial recruitments to MCA could be visualized nearby the distal M1 segment while occluding proximal-MCA with single clot initially. ${ }^{10}$ Given above factors, we chose to block M1 segment with two clots followed by ICA blockade. The cortex is supplied by terminal branches of MCA and lack of collateral recruitment, so we used the first clot to occlude distal M1 segment to produce ischemic lesions at the cerebral cortex, and the second clot to block proximal M1 segment, which will occlude lenticulostriate arteries or penetrating arteries supplied to this area and result in another lesion at basal ganglion. Also, using two clots with a total length of $10 \mathrm{~mm}$ could avoid thrombus fragmentation during injection efficiently. The angiograph confirmed that the intact clots were delivered into distal and proximal parts of M1 segment and leptomeningeal collateral recruitment scores were within a range of 3-5. Then, we blocked ICA with a catheter to reduce intracranial vascular dynamics and slow down clots autolysis. Thus, the infarct volumes were relatively stable and the degree of variability was $12.08 \%$, which was similar to what Rink et al ${ }^{2}$ reported.

Compared with Rink's results, ${ }^{2}$ the infarct volume in our study is smaller. The results obtained may probably be due to the following reasons: (1) intracranial vascular perforation and mechanical vasospasm are potential problems arising in an endovascular animal model. In their study, arteries were prone to undergo vasospasm in the process of catheterization through vertebrobasilar system and coils delivering; however, in our study, we just advanced the catheter into the proximal segment of left ICA without repeat catheterization; the sizes of the clots were suitable for MCA and then the clots generated proper radical force to the wall of the vessel. (2) They emphasized that angiographic evidence for pial collateral formation reconstituting the occluded territory was excluded from MR analysis. In our study, only three dogs with incomplete MCAO after procedure initially were excluded. The pial collateral supply with scores ranging from 3 to 5 to the MCA territory existed in the other dogs after complete MCAO. (3) A proper dose of heparin was given initially and neutralized after withdrawing the catheter in Rink's study. However, the 24-h survival rate of this coilinduced stroke models is relatively low. ${ }^{18}$ By comparison, it seems to be more important that animals that suffered from relatively larger infarcts could survive and be amenable for long-term study. Besides, evidence showed that patients with disabilities can benefit from highly specialized rehabilitation care and patient stroke care. ${ }^{4}$ As in our study, canine neurobehavioral deficits improved during the follow-up days accompanying with intensive care and restoration of blood flow. And, we could pay more attention to these questions in the following studies.

Recent revolutionary development of imaging requisition had made our approach to evaluate acute stroke by directly tracing the ischemic progress possible. Special MRI equipment was applied in the rodent stroke models. However, in this study, the equipment we used in the canine model is clinical 3.0-T MRI, which is similar to the one used in real clinical situations. MRI analysis provides the opportunity to measure accurately infarct volume of acute cerebral ischemia in vivo. ${ }^{2,13,18,28,29}$ In our study, we detected infarct $6 \mathrm{~h}$ after the procedure in all animals; all ischemic lesions were located in the basal ganglia and cerebral cortex. Infarct margins at $24 \mathrm{~h}$ after embolization were well-defined and the infarct volumes corrected for edema at $24 \mathrm{~h}$ after occlusion were correlated with leptomeningeal collateral scores and canine neurobehavioral scores, which were consistent with Christoforidis's findings. ${ }^{18}$ Usually, stroke was often described as an infarcted core surrounded by an ischemic penumbra. We found that there was a decrease in trend of the stroke volume along with the symptoms improvement from $24 \mathrm{~h}$ after embolization to the seventh day, which suggested that blood flow restore to the ischemic penumbra during the course needs further investigation. Furthermore, no hemorrhagic focus was identified in our model, which may correlate with the infarct volume and time point of MCA recanalization, ${ }^{30}$ and further observation will be needed. There was also no obvious cerebral edema or midline shift, which avoided the application of mannitol, which might disturb the nature course and lower the survival rate.

Some limitations that this study might have are: first, there was a learning course to navigate the canine cerebral vessels catheterization and deliver emboli in the pilot study. Second, considering maintaining nature course of acute phase of stroke model, we did not perform other serial angiograms to elucidate the precise time interval of complete MCA recanalization. Finally, we did not detect biomarkers of ischemic stroke, which may shed light on producing thrombolytic agents and therapeutic regimens.

The canine stroke model combined with autologous blood clots generated by MCAO and ipsilateral catheter-assisted ICA blockade reported here proved to be highly feasible and reproducible in beagles. Our findings indicated that the infarct volumes at $24 \mathrm{~h}$ after occlusion on T2WI were correlated with leptomeningeal collateral score negatively and canine neurobehavioral scores positively.

Supplementary Information accompanies the paper on the Laboratory Investigation website (http://www.laboratoryinvestigation.org)

\section{ACKNOWLEDGEMENTS}

We thank Andy Ai, MD, PhD, from the Department of Anesthesiology and Perioperative Medicine, UT MD Anderson Cancer Center (Austin, TX, USA) for his English editorial assistance. This work was supported by National Natural Science Foundation of China (30870710 to H-BS, and 81000653 to SL) and Medical Research Projects of Jiangsu province (RC2011074 to SL). 


\section{DISCLOSURE/CONFLICT OF INTEREST}

The authors declare no conflict of interest.

1. Shaibani A, Khawar S, Shin W, et al. First results in an MR imagingcompatible canine model of acute stroke. Am J Neuroradiol 2006; 27:1788-1793.

2. Rink C, Christoforidis G, Abduljalil A, et al. Minimally invasive neuroradiologic model of preclinical transient middle cerebralartery occlusion in canines. Proc Natl Acad Sci USA 2008;105:14100-14105.

3. Grunwald IQ, Wakhloo AK, Walter $\mathrm{S}$, et al. Endovascular stroke treatment today. Am J Neuroradiol 2011;32:238-243.

4. Mergenthaler P, Meisel A. Do stroke models model stroke. Dis Model Mech 2012;5:718-725.

5. Kang BT, Lee JH, Jung DI, et al. Canine model of ischemic stroke with permanent middle cerebral artery occlusion: clinical and histopathological findings. J Vet Sci 2007;8:369-376.

6. Ogun CO, Tastekin G, Kiresi $D$, et al. A new reversible ischemic neurologic deficit model in dogs. Med Sci Monit 2008;14:BR214-BR218.

7. Bailey EL, McCulloch J, Sudlow $C$, et al. Potential animal models of lacunar stroke: a systematic review. Stroke 2009;40:e451-e458.

8. Kang BT, Jang DP, Lee JH, et al. Detection of cerebral metabolites in a canine model of ischemic stroke using ${ }^{1} \mathrm{H}$ magnetic resonance spectroscopy. Res Vet Sci 2009;87:300-306.

9. Boulos AS, Deshaies EM, Dalfino JC, et al. Tamoxifen as an effective neuroprotectant in an endovascular canine model ofstroke. J Neurosurg 2011;114:1117-1126.

10. Liu S, Hu WX, Zu QQ, et al. A novel embolic stroke model resembling lacunar infarction following proximal middle cerebral artery occlusion in beagle dogs. J Neurosci Methods 2012;209:90-96.

11. Lee $\mathrm{KB}, \mathrm{Roh} \mathrm{H}$, Park HK , et al. Analysis of the lesion distributions and mechanism of acute middle cerebral artery infarctions involving the striatocapsular region. J Clin Neurol 2006;2:171-178.

12. Muir KW, Buchan A, von KR, et al. Imaging of acute stroke. Lancet Neurol 2006:5:755-768.

13. Harris AD, Kosior RK, Chen HS, et al. Evolution of hyperacute stroke over 6 hours using serial MR perfusion and diffusion maps. J Magn Reson Imaging 2009;29:1262-1270.

14. Hauck EF, Natarajan SK, Ohta $\mathrm{H}$, et al. Emergent endovascular recanalization for cervical internal carotid artery occlusion in patients presenting with acute stroke. Neurosurgery 2011;69:899-907, discussion 907.

15. Saarinen JT, Sillanpaa N, Rusanen $\mathrm{H}$, et al. The mid-M1 segment of the middle cerebral artery is a cutoff clot location for good outcome in intravenous thrombolysis. Eur J Neurol 2012;19:1121-1127.

16. Gerraty RP, Parsons MW, Barber PA, et al. Examining the lacunar hypothesis with diffusion and perfusion magnetic resonance imaging. Stroke 2002;33:2019-2024.
17. Lee KY, Latour LL, Luby M, et al. Distal hyperintense vessels on FLAIR: an MRI marker for collateral circulation in acute stroke. Neurology 2009;72:1134-1139.

18. Christoforidis GA, Rink C, Kontzialis MS, et al. An endovascular canine middle cerebral artery occlusion model for the study of leptomeningeal collateral recruitment. Invest Radiol 2011;46: 34-40.

19. Lipsanen A, Jolkkonen J. Experimental approaches to study functional recovery following cerebral ischemia. Cell Mol Life Sci 2011;68: 3007-3017.

20. Loubinoux I, Volk A, Borredon J, et al. Spreading of vasogenic edema and cytotoxic edema assessed by quantitative diffusion and T2 magnetic resonance imaging. Stroke 1997;28:419-426, discussion 426-427.

21. Niessen $F$, Hilger $T$, Hoehn $M$, et al. Differences in clot preparation determine outcome of recombinant tissue plasminogen activator treatment in experimental thromboembolic stroke. Stroke 2003;34: 2019-2024.

22. Molinari GF. Experimental cerebral infarction. I. Selective segmental occlusion ofintracranial arteries in the dog. Stroke 1970;1: 224-231.

23. Mchedlishvili $\mathrm{G}$, Kuridze $\mathrm{N}$. The modular organization of the pial arterial system in phylogeny. J Cereb Blood Flow Metab 1984;4:391-396.

24. Christoforidis GA, Mohammad Y, Kehagias D, et al. Angiographic assessment of pial collaterals as a prognostic indicator following intraarterial thrombolysis for acute ischemic stroke. Am J Neuroradiol 2005:26:1789-1797.

25. Gao H, Liu Y, Lu S, et al. A reversible middle cerebral artery occlusion model using intraluminal balloon technique in monkeys. J Stroke Cerebrovasc Dis 2006;15:202-208.

26. Liu Y, D'Arceuil HE, Westmoreland $\mathrm{S}$, et al. Serial diffusion tensor MR after transient and permanent cerebral ischemia in nonhuman primates. Stroke 2007;38:138-145.

27. Loftus CM, Greene GM, Detwiler KN, et al. Studies of collateral perfusion to canine middle cerebral artery territory. Am J Physiol 1990;259:H560-H566.

28. Rivers CS, Wardlaw JM, Armitage PA, et al. Acute ischemic stroke lesion measurement on diffusion-weighted imaging-important considerations in designing acute stroke trials with magnetic resonance imaging. J Stroke Cerebrovasc Dis 2007;16:64-70.

29. Kranz PG, Eastwood JD. Does diffusion-weighted imaging represent the ischemic core? An evidence-based systematic review. Am J Neuroradiol 2009;30:1206-1212.

30. Christoforidis GA, Karakasis C, Mohammad Y, et al. Predictors of hemorrhage following intra-arterial thrombolysis for acute ischemic stroke: the role of pial collateral formation. Am J Neuroradiol 2009; 30:165-170. 